\title{
Single Cell Sequencing of the Pineal Gland: The Next Chapter
}

OPEN ACCESS

Edited by:

James M. Olcese,

Florida State University, United States

Reviewed by:

Dragos Cretoiu,

Carol Davila University of Medicine and Pharmacy, Romania Yoav Gothilf,

Tel Aviv University, Israel

*Correspondence:

David C. Klein

kleind@mail.nih.gov

Specialty section:

This article was submitted to

Cellular Endocrinology,

a section of the journal

Frontiers in Endocrinology

Received: 28 June 2019

Accepted: 12 August 2019

Published: 20 September 2019

Citation:

Coon SL, FU C, Hartley SW, Holtzclaw L, Mays JC, Kelly MC Kelley MW, Mullikin JC, Rath MF, Savastano LE and Klein DC (2019) Single Cell Sequencing of the Pineal

Gland: The Next Chapter.

Front. Endocrinol. 10:590

doi: 10.3389/fendo.2019.00590

\author{
Steven L. Coon ${ }^{1}$, Cong Fu ${ }^{2,3}$, Steven W. Hartley ${ }^{4}$, Lynne Holtzclaw ${ }^{5}$, Joseph C. Mays ${ }^{6}$, \\ Michael C. Kelly ${ }^{7}$, Matthew W. Kelley ${ }^{8}$, James C. Mullikin ${ }^{9}$, Martin F. Rath ${ }^{10}$, \\ Luis E. Savastano ${ }^{11}$ and David C. Klein ${ }^{12 *}$
}

\begin{abstract}
${ }^{1}$ Molecular Genomics Core, Office of the Scientific Director, Eunice Kennedy Shriver National Institute of Child Health and Human Development, National Institutes of Health, Bethesda, MD, United States, ${ }^{2}$ Key Laboratory of Organ Regeneration \& Transplantation of the Ministry of Education, The First Hospital of Jilin University, Changchun, China, ${ }^{3}$ National-Local Joint Engineering Laboratory of Animal Models for Human Diseases, Changchun, China, ${ }^{4}$ Comparative Genomics Analysis Unit, Cancer Genetics and Comparative Genomics Branch, National Human Genome Research Institute, National Institutes of Health, Bethesda, MD, United States, ${ }^{5}$ Microscopy and Imaging Core, Office of the Scientific Director, Intramural Research Program, Eunice Kennedy Shriver National Institute of Child Health and Human Development, National Institutes of Health, Bethesda, MD, United States, ${ }^{6}$ Institute on Systems Genetics, New York University School of Medicine, New York, NY, United States, ${ }^{7}$ Single Cell Analysis Facility, Frederick National Lab for Cancer Research, National Cancer Institute, National Institutes of Health, Bethesda, MD, United States, ${ }^{8}$ Section on Developmental Neuroscience, Laboratory of Cochlear Development, Division of Intramural Research, National Institute on Deafness and Other Communication Disorders, National Institutes of Health, Bethesda, MD, United States, ${ }^{9}$ National Institutes of Health Intramural Sequencing Center, National Human Genome Research Institute, National Institutes of Health, Rockville, MD, United States, ${ }^{10}$ Department of Neuroscience, Panum Institute, University of Copenhagen, Copenhagen, Denmark, ${ }^{11}$ Department of Neurosurgery, University of Michigan, Ann Arbor, MI, United States, ${ }^{12}$ Office of the Scientific Director, Intramural Research Program, Eunice Kennedy Shriver National Institute of Child Health and Human Development, National Institutes of Health, Bethesda, MD, United States
\end{abstract}

The analysis of pineal cell biology has undergone remarkable development as techniques have become available which allow for sequencing of entire transcriptomes and, most recently, the sequencing of the transcriptome of individual cells. Identification of at least nine distinct cell types in the rat pineal gland has been made possible, allowing identification of the precise cells of origin and expression of transcripts for the first time. Here the history and current state of knowledge generated by these transcriptomic efforts is reviewed, with emphasis on the insights suggested by the findings.

Keywords: pineal, single cell sequencing, melatonin, transcriptomics, adrenergic, transcriptome profiling

\section{INTRODUCTION}

The pineal gland is composed $90-95 \%$ of pinealocytes, which synthesize melatonin (1). Studies of the pineal gland have addressed the levels of transcripts involved in this process and have experienced remarkable improvements, innovations, and enhancements, in parallel with advances in cell biological techniques that have characterized the field. In general, genes expressed exclusively in non-pinealocytes have been ignored.

The first efforts to study a single mRNA transcript in the pineal gland came from northern blot analysis in the late 1980s (2-5). It required the equivalent of several rat pineal glands (4-5 mg wet weight); RNA was extracted, electrophoresed and blotted. This allowed for the radiochemical detection of transcripts encoding tryptophan hydroxylase1 (Tph1) and acetylserotonin methyltransferase $($ Asmt $)$ /hydroxyindole-O-methyltransferase, the transcripts that encode the first and last enzymes in melatonin synthesis, respectively. The northern blot technique was highly useful, especially because it allowed the resolution of distinct molecular species. 
However, it was obviously limited by the amount of tissue required and the small number of transcripts it could detect on repeated stripping and probing of blots.

The reverse transcription polymerase chain reaction was introduced into the pineal literature early in the 1990s (613). It was highly popular because it was sensitive and allowed multiple transcripts to be measured using small amounts of mRNA. It was used to detect low levels of transcripts including receptors and clock genes. However, quantitation with the method was somewhat unreliable and results could only reflect changes in small regions of mRNA amplified by the technique, which permits off-target results and precludes examination of the entire transcripts, which may have reflected gene leakage. Another problem with PCR was overamplification of very weakly expressed transcripts. In addition, analysis of each transcript was hands-on, limiting the number of transcripts that could be detected on a routine basis.

A revolutionary method was introduced to pineal cell biology with cDNA arrays, which at the start allowed for the detection of several hundred targets (14) and ultimately developed into microarrays, which permitted thousands of targets to be probed simultaneously using as little as one rat pineal gland (15-19) or 10 larval zebrafish pineal glands (20). However, this technique had the disadvantage of probing only portions of a transcript and was only useful for those transcripts which were represented on the microarray chip. Putting aside these limitations, this technique made important advances by reducing the amount of tissue required and increasing the number of genes probed. In the case of the rat pineal gland, it revealed large day/night changes in hundreds of transcripts, many more than had been realized at the time (19). The technique was also useful in comparing the pineal gland and retina and in determining the large number of genes shared by these two tissues.

The limitations of the cDNA chip technology were rapidly overcome in the early years of this century with the development of methods that sequenced the entire transcriptome, also referred to as bulk sequencing. Sequences have been obtained for chicken, human, mouse, rat, rhesus, and zebrafish pineal glands (21-30). This provided the sequence of full length transcripts, including the coding and flanking regions. It also provided an indication of splicing and alternative polyA sites. It sequenced all transcripts, known and unknown, including noncoding long and short RNAs $(21,29,31)$. The technique is remarkably sensitive, allowing for tens of thousands of transcripts to be sequenced with the mRNA from a fraction of a single rat pineal gland.

As applied to the pineal gland, this technique provided excellent data on day/night differences. Moreover, studies on the rat pineal gland have provided valuable information on the effects of superior cervical ganglionectomy (SCGX) or decentralization (DCN) in in vivo experiments, and the effects of norepinephrine or dibutyryl cyclic AMP in in vitro experiments (Figure 1) (25). These confirmed and expanded previous results on the rat pineal gland, which showed that there was a broad change in the transcriptome on a 24 -h basis. It also showed that neural stimulation of this tissue, in the form of postganglionic projections from the superior cervical ganglia stimulated the gland, based on the observation that both forms of surgical denervation, SCGX and DCN, blocked these changes. In addition, it revealed that most of these changes could be driven in vitro by norepinephrine or by its second messenger cyclic AMP. It is noteworthy that comparison of the transcripts that were induced more than 4 -fold at night and by norepinephrine or dibutyryl cyclic AMP were nearly identical, numbering about 50 [Table S1 in (25)]. This correlation supported the view that the day/night differences were driven by a norepinephrine-cyclic AMP mechanism. It should be noted that the correlation was lower with weakly induced genes, which may be a reflection of statistical variation.

The development of advanced sequencing methods has evolved and deserves brief mention here. A hybrid approach is now available that combines Illumina short-read/highthroughput RNA-Seq with targeted qPCR and long-read Pacific Biosciences SMRT sequencing. In pineal gland studies it has been possible to identify 20 alternative RNA isoforms of the Ttc8/BBS8 gene (23). This gene was known to exist in multiple isoforms and is of interest because of evidence that it is involved in the Bardet-Biedl syndrome and non-syndromic retinitis pigmentosa (32-35). This technique is severely limited by the number of genes it can detect on a practical basis, but holds great promise for the study of isoforms, a complex and difficult endeavor. The interested reader is referred to the original publication for more details (23).

The most recent advance in sequencing is single cell RNA sequencing (scRNA-seq) (36). It takes several forms all of which allow for thousands of single cells to be sequenced simultaneously, yielding several thousand transcripts per cell. Overall, the technique has extremely high sensitivity and generates an enormous amount of data on the transcriptomes expressed in individual cells.

The technique was introduced into the pineal literature because of the suggestion that there were two populations of the cell that were defined by large differences in ASMT protein (37). As mentioned above, ASMT is the last enzyme in melatonin synthesis and converts the melatonin precursor $\mathrm{N}$ acetylserotonin to melatonin. We hoped that the new technology would provide a transcriptional profile of each cell type and answer the question of whether pinealocyte subtypes defined by different levels of ASMT exist.

\section{SINGLE CELL RNA SEQUENCING}

\section{Cell Isolation}

The isolation of single pineal cells followed a wellestablished method, which has been used for biochemical, electrophysiological, and cytochemical studies (38-42). Glands were removed, soaked in DMEM solution and then cleaned under a microscope to limit the contaminating cells coming from blood and connective tissue. The glands were then placed in a freshly prepared Papain Dissociation System (Worthington; Lakewood, NJ) containing DNAase; details of the procedure have been published (43). 


\section{In vivo Analyses}

\section{In vitro Analyses}
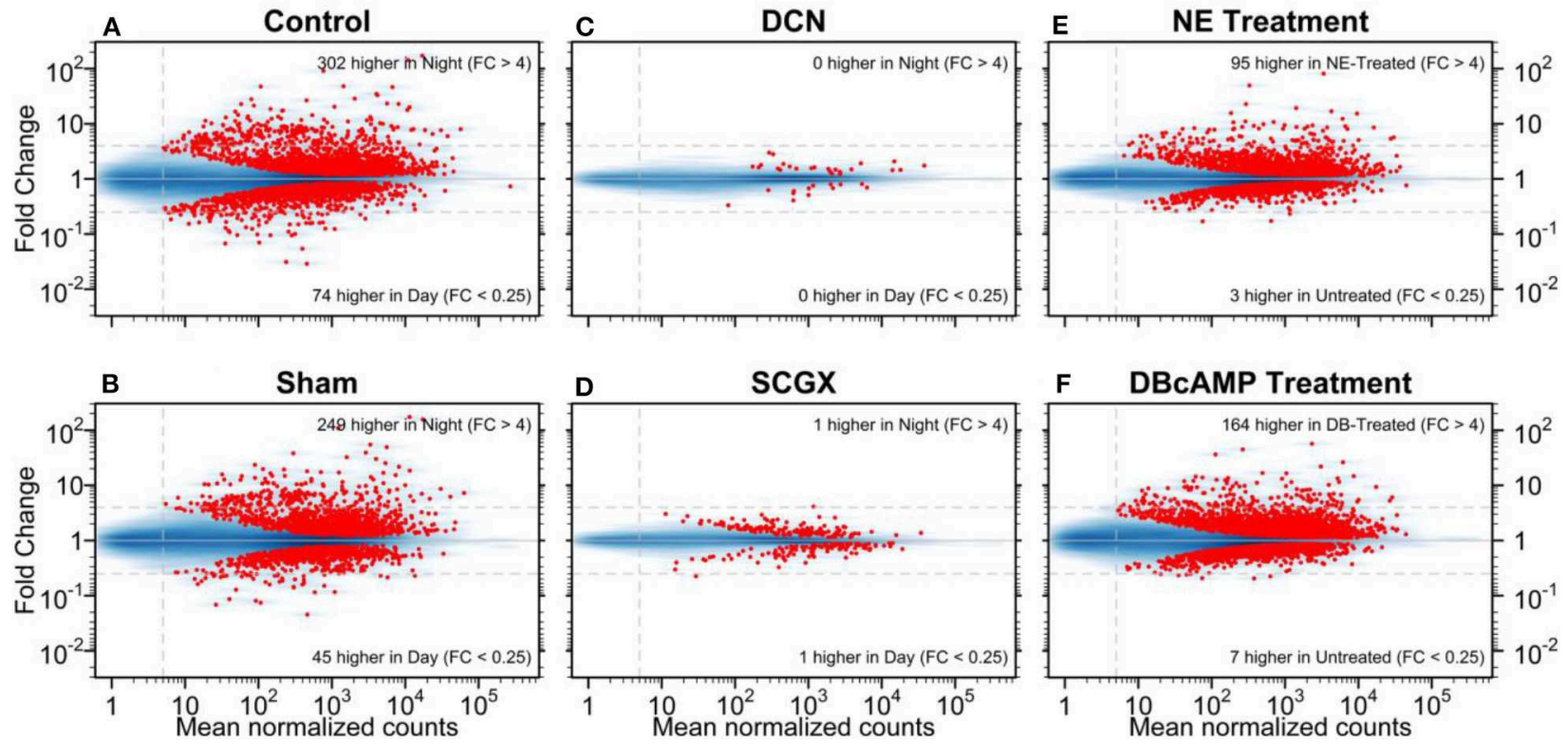

FIGURE 1 | Day/night differences in gene expression. The mean normalized read-pair counts (x-axis) vs. the estimated fold change (y-axis) are displayed on a log-log scale. Four in vivo and two in vitro analyses are presented as MA plots. The blue shading indicates the density of genes, and each red point represents a gene with statistically significant differential expression (adjusted- $p<0.001$ ). Dashed horizontal lines mark 4-fold changes in both directions; the dashed vertical line indicates the minimum abundance threshold for the statistical tests. The four in vivo analyses compared night and day time points in adult rats for the following groups: (A) no surgery (Control); (B) neonatal sham surgery (Sham); (C) neonatal superior cervical ganglia decentralization (DCN); (D) neonatal superior cervical ganglionectomy (SCGX). The two in vitro analyses compared treated/untreated pineal glands: (E) norepinephrine-treated (NE) vs. untreated and (F) dibutyryl-cyclic-AMP-treated (DBcAMP) vs untreated. Reproduced from Hartley et al. (25). This figure and associated legend is published with permission of the original publisher under license CC0.1.0.

\section{Single Cell Analysis}

Single-cell cDNA libraries were constructed using a Chromium Controller (10X Genomics; Pleasanton, CA) and the Chromium Single Cell 3' Reagent Kits v2 (43). In brief, dilute solutions of completely dissociated preparations of single cells were introduced into a stream of oil to make microdroplets. Sequencing reagents in the stream included a "sponge" that contains a unique cDNA marker for identification of the cell source of each transcript: one marker-one cell. This unique cDNA marker was incorporated into the mRNA at the polyA end, thereby providing a means of tracking the originating cell source of each molecule.

The microdroplets containing individual cells were mixed together and sequenced (Illumina HiSeq2500, Illumina; San Diego, CA). Ninety-eight bp sequences were produced in close proximity to the polyA tails. It was possible to recover 2,4004,300 cells per sample, with $40-70 \mathrm{k}$ reads per cell and 2,7003,000 genes per cell detected on average (43).

The analysis of sequenced single-cell libraries was done by generating gene-level counts with the CellRanger analysis software v2.1.0 (10X Genomics). This aligns sequencing reads to the rat Rnor6.0 reference genome (Ensembl). The sequenced cells were subsequently filtered to remove doublets and low abundance genes. Dimensional reduction analysis was done (Seurat v2.2.0 package for R). Gene counts were normalized to
$10^{4}$ molecules per cell. Lists of $\sim 1,500$ highly variable genes for the day and the night samples were prepared and used to compute principal components (PC) using RunPCA; the results of $\mathrm{PC}$ analysis were projected onto the remaining genes with ProjectPCA (43).

The clustering of cells was done by employing a shared nearest neighbor (SNN)-based algorithm; results were imaged by t-distributed stochastic neighbor embedding (t-SNE) through RunTSNE (parameters: do.fast $=$ TRUE). The $2 \mathrm{D}$ projections of the cells generated by this method generates clusters that are color-coded according to FindClusters output. The identity of the clusters were determined using known marker genes. In each sample, the $\beta$-pinealocyte population was embedded on the $\mathrm{t}$ SNE plot as a single cluster; it was divided into smaller colorcoded clusters by the SNN clustering algorithm. These clusters were consolidated into one large cluster for subsequent study to match the t-SNE embedding. Cellular doublets were eliminated based on expression of moderate-to-high levels of genes that were markers for separate clusters (day, $n=60$; night, $n=125$ ) (see reference (43) for additional details).

\section{NINE CELL TYPES OF THE PINEAL GLAND}

Over 5,000 individual cells were subjected to cluster analysis, which detected five major cell types: pinealocytes, astrocytes, 
microglia, vascular and leptomeningeal cells (VLMCs), and endothelial cells (Figure 2A). The expression of marker genes in these cells confirmed this finding. It was possible to further

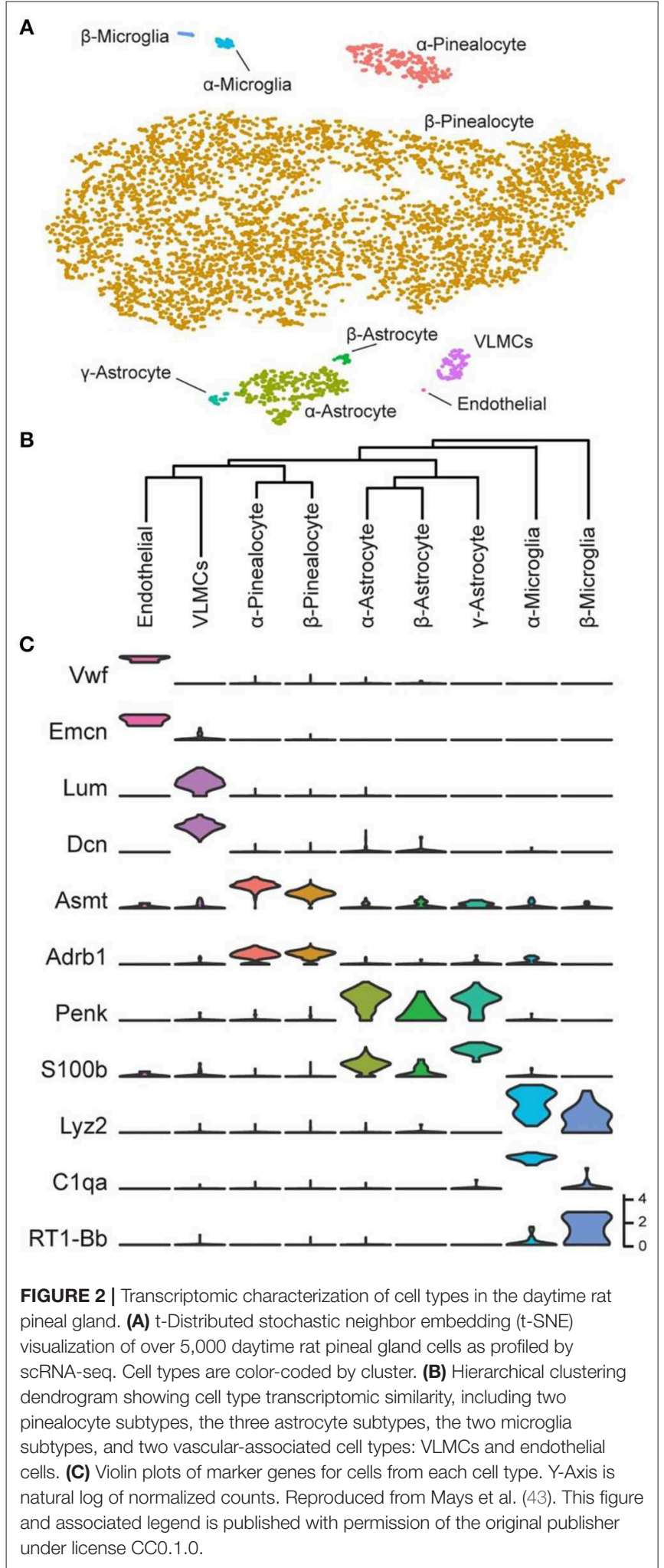

resolve the cell types according to the results of cluster analysis and marker gene abundance into two pinealocyte subypes ( $\alpha$ and $\beta$ ), three subtypes of astrocytes $(\alpha, \beta$, and $\gamma)$, and two subtypes of microglia ( $\alpha$ and $\beta$ ). Transcriptomic relationships of the nine cell types as indicated by hierarchical clustering are consistent with our assigned designations (Figure 2B).

\section{Pinealocytes}

The precise proportion of cell-types is difficult to determine with a high degree of confidence because of differences in recovery and cell stability during isolation. However, with this limitation in mind, it appears that $90 \%$ of the profiled cells were pinealocytes [Table S1 in (43)], which is generally in line with morphological studies $(1,44)$ (Figure 3A). These cells all expressed high levels of Tph1, Asmt, and Sag [Figure 2C; Figure S1 in (43)]. These cells also expressed high levels of Gngt1, Gngt2, Rom1, Crx, Cngb1, Cnga1, Pde6c, and Slc6a6; receptors for adrenergic agonists $A d r b 1, A d r a 1 b$, and Drd4; and, receptors for cholinergic agonists Chrna3 and Chrnb4 [Figure 2C; Figures S1, S2 in (43)]. In addition, these cells expressed a group of 49 transcripts found nearly exclusively in the pineal gland and retina (19) including Sag [Figure S1 in (43)], Gngt1 and Gngt2 [Figure S4 in (43)], Crx and Neurod1 [Figure S19 in (43)], Pde6b [Figure S15 in (43)], Drd4 [Figure S2 in (43)], and Cacnalf, Cnga1, and Cngb1 [Figure S13 in (43)]. The expression of these transcripts exclusively in pinealocytes has not been directly demonstrated previously in most cases; this is because a homogenized mixture of cells in the pineal gland had been used in earlier bulk sequencing

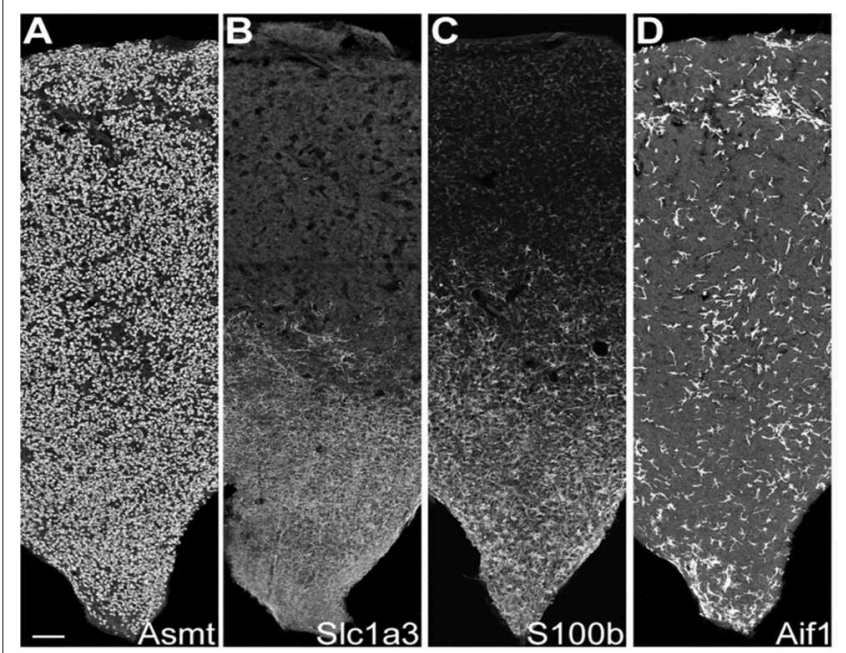

FIGURE 3 | IHC reveals cell type-specific patterns of expression. IHC sections through the rat pineal gland midline; rostral stalk origin at the bottom. The length and middle third of the width of the gland appear. Scale bar $=100 \mu \mathrm{m}$. (A) Uniform distribution of ASMT-positive pinealocytes. (B) Slc1a3-positive $\gamma$-astrocytes abundance is greatest in rostral/stalk region. (C) S100b-positive cells are abundant in the rostral region; they appear elsewhere with distinctly lower density and weaker expression strength. (D) Aif1-positive cells are unevenly distributed throughout pineal gland at low density. See Figure S6 in Mays et al. (43) for full images and further details. Reproduced from Mays et al. (43). This figure and associated legend is published with permission of the original publisher under license CC0.1.0. 
A
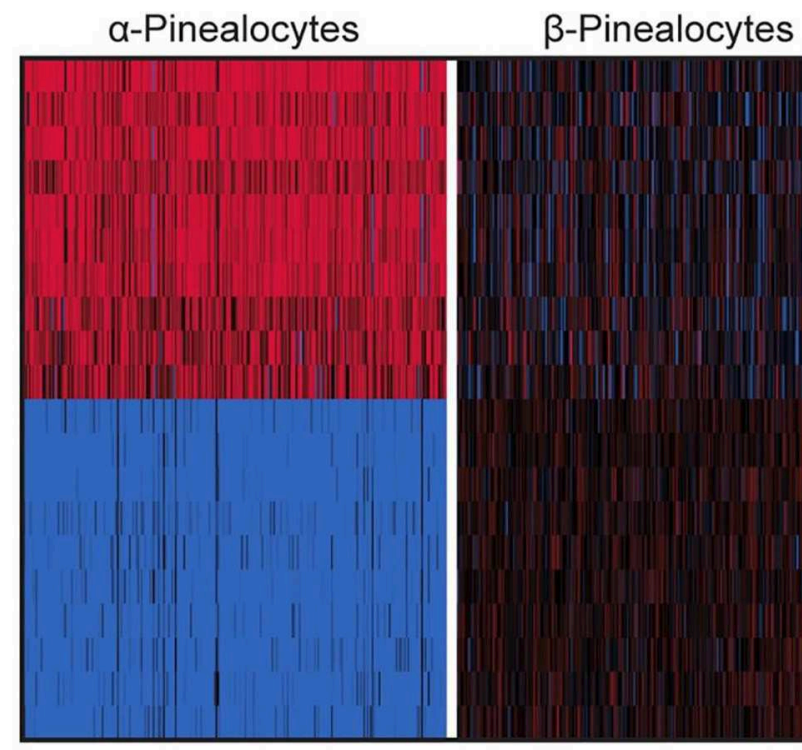

B

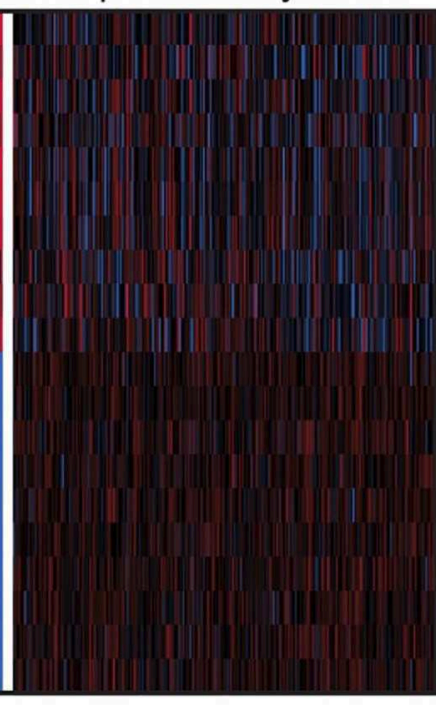

Mt-cox3

Clu

Mt-atp6

Psap

Mt-cyb

Mt-co2

Mt-nd1

Lgmn

Asmt

Ubc

Rpl19

Rpl41

Gpx4

LOC687780

High

Rps 14

Rps 16

Tma7

Rplp1

Rps11

Rpl35

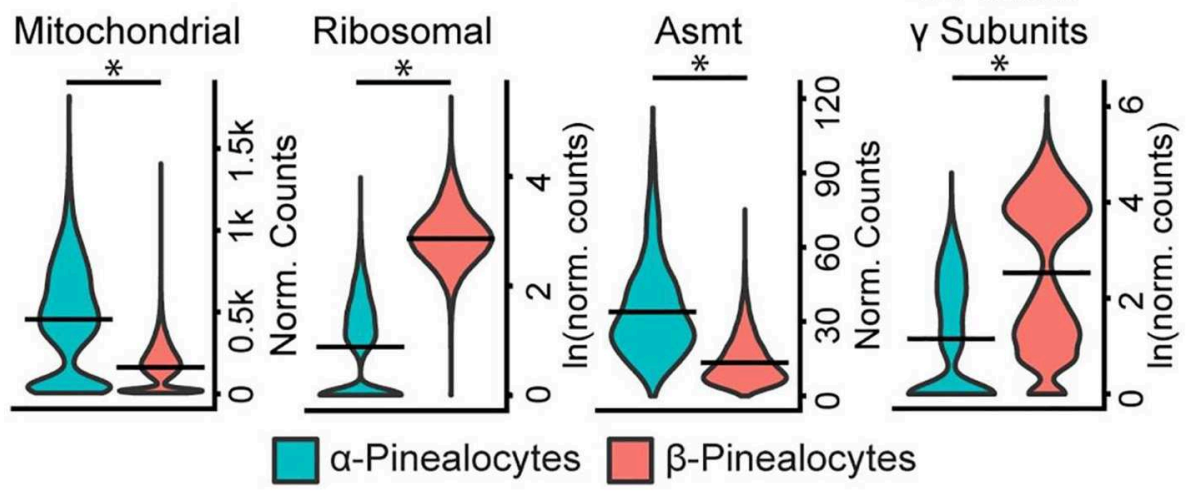

FIGURE 4 | scRNA-seq reveals two transcriptionally distinct pinealocyte populations. (A) A heatmap of expression values for the top 10 most differentially expressed genes (by effect size) for $\alpha$ - and $\beta$-pinealocytes. Values are Z-scores of counts calculated between all cells of both cell types. Each column represents one cell: random samples of 250 cells per cell type are presented. (B) Violin plots of expression distribution differences between two pinealocyte subtypes for three functional groups and one gene, Asmt. Y-Axis is either normalized counts or natural log (In) of normalized counts. Horizontal lines represent the mean. * $p<0.001$, Wilcoxon rank sum test. All cells from each subtype are included ( $\alpha$-pinealocyte, $n=275 ; \beta$-pinealocyte, $n=4,822$ ). Mitochondrial group includes differentially expressed mitochondrial OxPhos genes ( $p<0.05, N=12$, fold change $\geq 2.0$ ), ribosomal group includes top 20 most differential ribosomal genes by effect size ( $p<0.05$, fold change $\geq 2.0$ ), G-protein $\gamma$-subunits include Gngt1, Gngt2, Gng10, and Gng13 [see Figure S5 in (43) for individual genes]. Reproduced from Mays et al. (43). This figure and associated legend is published with permission of the original publisher under license CC0.1.0.

studies, precluding the clear association of a gene with a cell type. However, several lines of evidence in the literature point to this conclusion (43).

Five percent of pinealocytes were the $\alpha$-subtype; the remaining pinealocytes were $\beta$-subtypes. Although these two cell types share a characteristic set of marker genes and function as sources of melatonin, analysis revealed some distinct genetic differences and differences in sets of functional groups. The most outstanding included (1) Asmt; (2) mitochondrial oxidative phosphorylation (OxPhos) genes; (3) genes that comprise the ribosomal genome; and, (4) G-protein $\gamma$-subunits [Figure 4A; Figures S1, S3, S4 in (43)]. Asmt expression (Figure 4B) in $\alpha$-pinealocytes was 3.4 -fold greater, supporting results from previous immunohistochemical studies of ASMT protein (37). The counts for OxPhos and ribosomal transcriptomes were pooled [Figure S5 in (43)] for analysis; $\alpha$-pinealocytes had a 2.3fold greater average expression of eight differentially expressed OxPhos genes, and 8.2-fold lower expression of the top 20 differentially expressed ribosomal genes. There also is a 5.4-fold lower average expression of G-protein $\gamma$-subunits Gngt1, Gngt2, Gngt10, and Gng13 in $\alpha$-pinealocytes relative to $\beta$-pinealocytes [Figure 4B; Figure S4 in (43)]. The possibility that $\alpha$-pinealocytes represent stressed cells was rejected because of the opposite and robust differences between the levels of OxPhos and ribosomal genes: the former being higher in $\alpha$-pinealocytes and the latter higher in $\beta$-pinealocytes. 

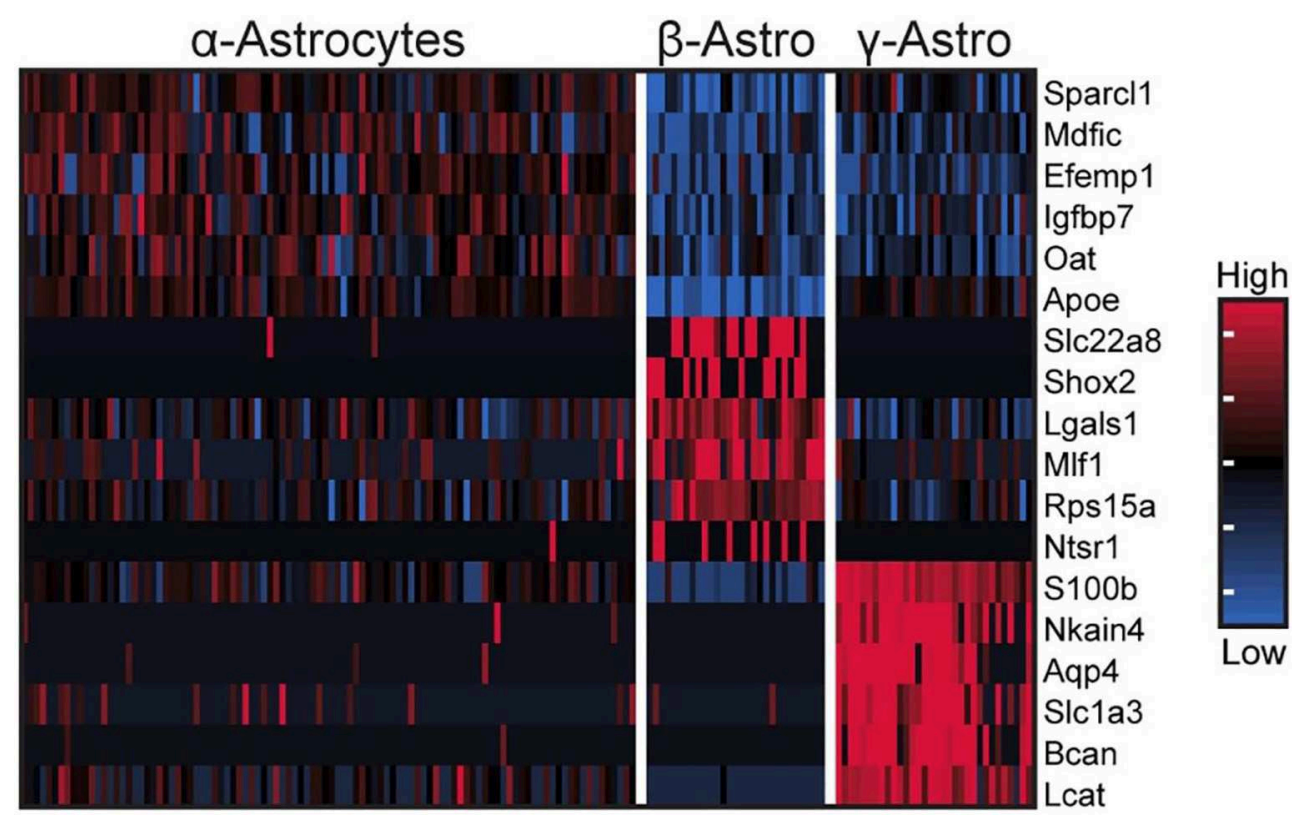

FIGURE 5 | scRNA-seq reveals three transcriptionally distinct astrocyte populations. Heatmap of expression values for the top 6 highest differentially expressed genes (by effect size) for $\alpha$-, $\beta$-, and $\gamma$-astrocytes. Values are Z-scores of counts calculated between all cells of the three cell types. Each column represents one cell; a random sample of 100 cells from $\alpha$-astrocytes are shown; all $\beta$ - and $\gamma$-astrocytes are shown. See also Figure S1 in Mays et al. (43) for more information. Reproduced from Mays et al. (43). This figure and associated legend is published with permission of the original publisher under license CC0.1.0.

\section{Astrocytes}

These cells accounted for seven percent of the cells [Table S1 in (43)], based on expression of known markers including Aldh1a1, S100b, and Tnfrsf21 [Figure 2C; Figure S1 in (43)] (45-47). These cells also highly expressed Penk, Apoe, and Esm1 [Figure 2C; Figure S1 in (43)]. The percentages of $\alpha$-, $\beta$-, and $\gamma$-astrocytes were 85,7 , and $8 \%$, respectively. $\alpha$-Astrocytes had higher Sparcl1, Mdfic, Efemp1, Oat, and Gad2 expression relative to other subtypes. The $\beta$-astrocytes expressed Slc22a8, Shox2, Lgals1, and Mlf1 at higher levels than other subtypes. $\gamma$ Astrocytes were characterized by stronger expression of $S 100 \mathrm{~b}$, Nkain4, Aqp4, Slc1a3, Bcan, and Gfap [Figure 5; Figure S1 in (43)]. Histochemical analysis revealed that $\gamma$-astrocytes were primarily limited in distribution to the pineal stalk region, as indicated by Slc1a3 [Figure 3B; Figure S6B (43)], as was true of Gfap protein [Figures S6C, S7C in (43)]; this is consistent with previous observations (48-50). $S 100 b$ was expressed in all astrocyte subtypes, but was strongest in $\gamma$-astrocytes [Figure 5; Figure S1 in (43)]. Detection of S100b-postive cells by IHC revealed astrocytes occur throughout the gland, though higher expression was present in the pineal stalk region, consistent with the higher expression of $S 100 b$ exhibited by $\gamma$-astrocytes [Figure 3C; Figures S6D, S7D in (43)].

\section{Microglia}

One percent of the profiled cells [Table S1 in (43)] were classified as microglia according to expression of Aif1 and Lyz2 [Figure 2C; Figure S8 in (43)] (45-47). AIF1 IHC Positive cells were present throughout the gland [Figure 3D; Figure S6E in
(43)]. $\alpha$ - and $\beta$-microglia subtypes comprised 64 and $36 \%$ of microglia, respectively. These cells were strongly differentiated by complement components Clqa, Clqb, and Clqc, which were high in $\alpha$-microglia. $\beta$-Microglia in contrast had low levels of the complement component transcripts, but high levels of MHC Class II transcripts $R T 1-D a, R T 1-D b 1$, and $R T 1-B a$ [Figure S8 in (43)].

\section{Vascular Cells}

Endothelial cells and VLMCs were detected in low abundance. These cells appear to be in intimate contact, based on expression of Cdh11 and Gja1 in both [Figure S10 in (43)]. Endothelial cells accounted for $0.1 \%$ of cells profiled and were characterized by the expression of $V w f$, Emcn, and other markers (43).

VLMCs were $2 \%$ of the profiled cells, identified by expression markers Lum, Dcn, Colla1, and Gjb2 [Figure 2C; Figure S9 in (43)] (51). VLMCs have never been described in the pineal gland prior to this study. They have the potential of major importance in acting as mediators between circulating signals and pineal cells, in addition to contributing to the extracellular matrix reflecting the expression of collagen and extracellular matrix proteins.

For example, they have receptors for circulating ligands which could act to alter the synthesis and release of secondary signals that impact the function of other cells in the gland. Pineal VLMCs exclusively express Il13ra2 [Figure S9 in (43)], the transcript that encodes a selective receptor for the cytokine interleukin Il13 (52). Interaction of Il13 with its receptor could impact the pineal gland broadly, perhaps through effects on the extracellular matrix. In addition, vascular cells could act on pinealocytes and astroctyes 


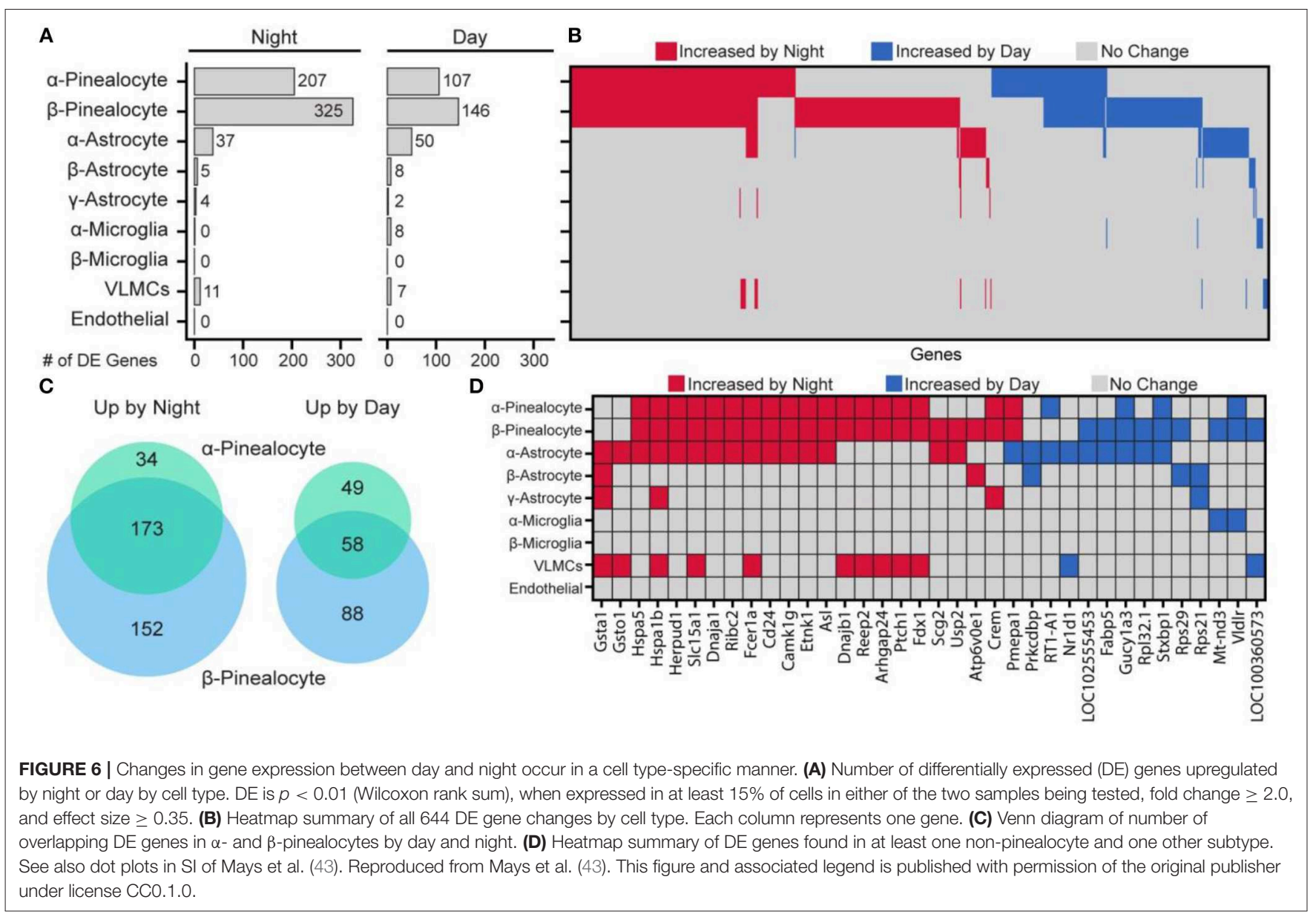

through contact-dependent ephrin ligand-receptor mechanisms (53). Specifically, the ephrin ligand EFNA1 on endothelial cells could bind to the ephrin receptor EPHA4 on pinealocytes; and, the ligand EFNB1 on VLMCs could bind to the receptor EPHB1 on astrocytes (Figure S17 in (43)]. It should also be mentioned that, VLMCs are a standout among cells in the pineal gland for expression of the $\alpha_{2 \mathrm{~A}}$-adrenergic receptor, encoded by Adra $2 a$ [Figure S2 in (43)]. Activation of this receptor by circulating norepinephrine or epinephrine can cause catecholamine-induced inhibition of adenylate cyclase and as a result inhibit cyclic AMP dependent processes in the VLMCs. Although of interest, it should be noted that the above are speculations and accordingly require further study to determine their relevance in the context of pineal cell biology.

\section{DAY AND NIGHT CHANGES IN THE PINEAL CELL TRANSCRIPTOME}

Day and night expression values in specific pineal cell types were compared. There were considerable differences among the cell subtypes in the number of genes that were differentially expressed between day and night (Figure 6). The largest differential expression was found in pinealocytes: 359 genes were upregulated at night and 195 genes were upregulated during daytime. Consistent with prior studies, differentially upregulated genes included Aanat, Crem, Drd4, Pde10a (19, 25). Overall, $\beta$ pinealocytes had 1.5 -fold more genes differentially expressed than $\alpha$-pinealocytes, with considerable overlap: 173 and 58 of the same transcripts were increased in both subtypes during night and day, respectively (Figure 6C). Non-pinealocytes had generally lower day/night differential expression, with the $\alpha$ astrocytes having 37 genes higher at night and 50 higher during the day. There were relatively fewer differentially expressed transcripts in other non-pinealocytes several of which overlapped between different cell types (Figure 6D). The molecular basis of changes in astrocytes is not clear. Whereas, $\alpha$ - and $\beta$-adrenergic mechanisms control changes in pinealocytes, the responsible receptors are absent from astrocytes. Other receptors might mediate these changes. Alternatively, the day/night differences could reflect the functioning of an internal clock in these cells, although expression of clock genes is not high [Figure S22 in (43)].

Pinealocytes have a high amount of Aanat at night. Aanat transcripts were also detected at uniformly low levels in non-pinealocytes [Figure S1 in (43)], probably due to contamination by pinealocyte-derived ambient mRNA. This results in non-pinealocytes erroneously seeming to express Aanat 


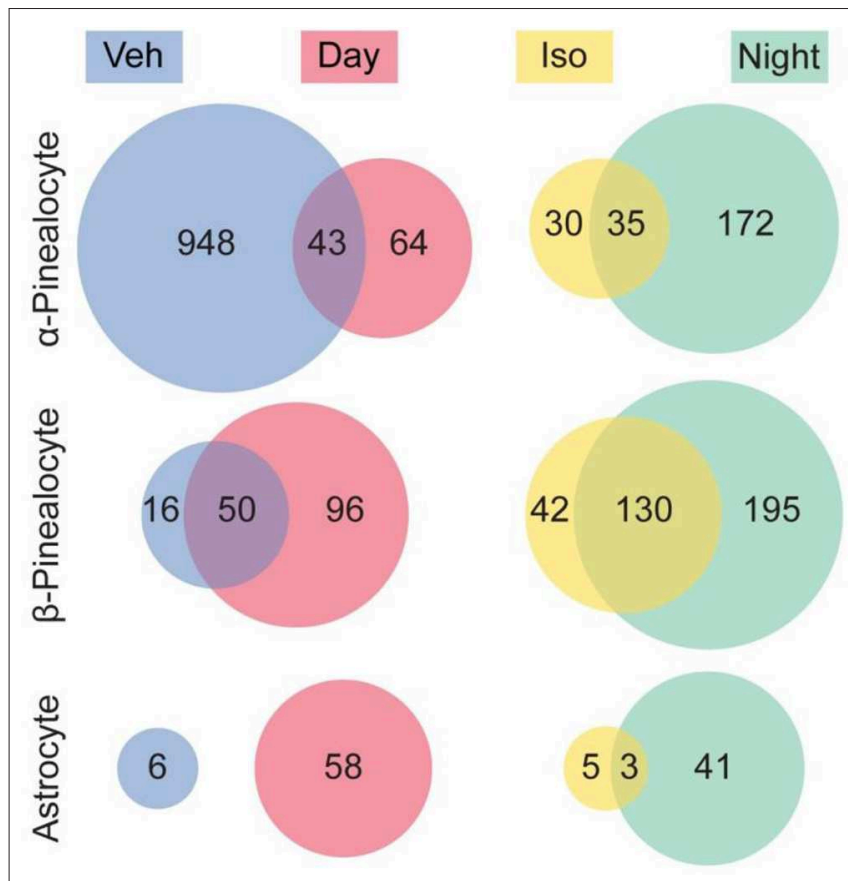

FIGURE 7 | Comparison of differentially expressed genes between the night time pineal gland and isoproterenol-treated pineal gland. Venn diagrams indicate the number of genes that were significantly differentially expressed (DE) in the pineal gland. There was overlap between genes exhibiting DE upregulated at night and by isoproterenol (Iso) treatment, as well as overlap between genes exhibiting DE upregulated during the day and upregulated in the vehicle control treated (i.e., downregulated by isoproterenol treatment), in 3 cell types. Other cell types are not shown. Reproduced from Mays et al. (43). This figure and associated legend is published with permission of the original publisher under license CC0.1.0.

differentially. Because of this, the gene was deleted from nonpinealocyte analysis. Pmepal was determined to be unusual because it was upregulated at night in one cell type ( $\alpha$ - and $\beta$-pinealocytes) but upregulated during the day in $\alpha$-astrocytes (Figure 6D).

\section{ISOPROTERENOL TREATMENT MIMICS DAY/NIGHT CHANGES IN THE PINEAL CELL TRANSCRIPTOMES OF PINEALOCYTES}

It is known that treatment with the $\beta$-adrenergic agonist isoproterenol during daytime has similar effects on the pineal transcriptome to those that occur due to neural stimulation at night. It is used in place of norepinephrine because isoproterenol is not taken up into nerve endings in the pineal perivascular space, whereas norepinephrine is rapidly and selectively taken up, thereby largely preventing adrenergic activation (54).

The results of our studies were in line with the interpretation that $97 \%$ of the transcriptional changes observed following isoproterenol treatment were in $\alpha$ - and $\beta$-pinealocytes (Figure 7). This is in agreement with findings of high enrichment with $\beta$-adrenergic receptors that mediate night time changes in gene expression. Astrocytes had the remaining 3\% of changes, whereas changes in other cells were not detected. It should be noted that upregulation of a similar number of the same genes was observed in isoproterenol-treated glands and in night time glands: upregulation of 54,76 , and $38 \%$ of the same genes was seen in $\alpha$-pinealocytes, $\beta$-pinealocytes, and astrocytes, respectively. Four and seventy-six percent of genes suppressed due to isoproterenol treatment in $\alpha$-pinealocytes and $\beta$-pinealocytes, respectively, were also suppressed during the day, that is, they appeared to be upregulated after vehicle control treatment.

\section{IMPLICATIONS}

There are broad implications of the findings of scRNA-seq analysis. Several points of interest can be identified, including the selective mechanisms involved in astrocyte gene expression. However, the feature which is especially worthy of additional comment here is the finding of two pinealocyte subtypes. As discussed above, $\alpha$-pinealocytes are characterized by high levels of Asmt and high levels of the mitochondrial genome, and low levels of protein synthesis transcripts and Gngt1 and Gngt2, in contrast to the more abundant $\beta$-pinealocytes (Figure 4B). Together, they are responsible for the synthesis of melatonin in the pineal gland, with slightly different roles.

It is proposed that the $\alpha$-pinealocytes are especially highly adapted for the last step in melatonin synthesis. This is supported not only by the high levels of Asmt but by the accompanying increase in ATP production by the OxPhos pathway. The main impact on melatonin synthesis is that high ATP leads directly to an increase in SAM, which is synthesized from ATP and methionine (Figure 8). Thus, the cells containing both these effects are in a position to methylate $\mathrm{N}$-acetylserotonin at high levels. The focus of the cells on melatonin synthesis is further evidenced by the low levels of protein synthesis enzymes; protein synthesis is the primary consumer of cellular ATP. Adding to this are the low levels of Gngt1 and Gngt2, indicating that G-protein based signal transduction is suppressed; this is in agreement with the finding of lower levels of gene induction in these cells, as discussed above.

The existence of two functionally different pinealocyte subtypes raises the issue of whether $\alpha$-pinealocytes are compromised to a degree that interferes with the functioning of these cells. This could occur due to relatively lower metabolites and suppressed protein synthesis. One can argue that the absence of some functions, such as maintenance of extracellular matrix, would be compensated for by $\beta$-pinealocytes. Also, some essential factors that are reduced in the $\alpha$-pinealocytes could be provided by the $\beta$-pinealocyte. Cell:cell transfer of these factors via gap junctions, membrane permeability, and import/export mechanisms might mediate this export:import function. Also, some proteins may have sufficient stability to prevent a loss of function. Moreover, lowered activity of some processes in the $\alpha$-pinealocytes may enhance ASMT activity by lowering the production of inhibitors and producing a more favorable biochemical environment for ASMT. Hence, it 


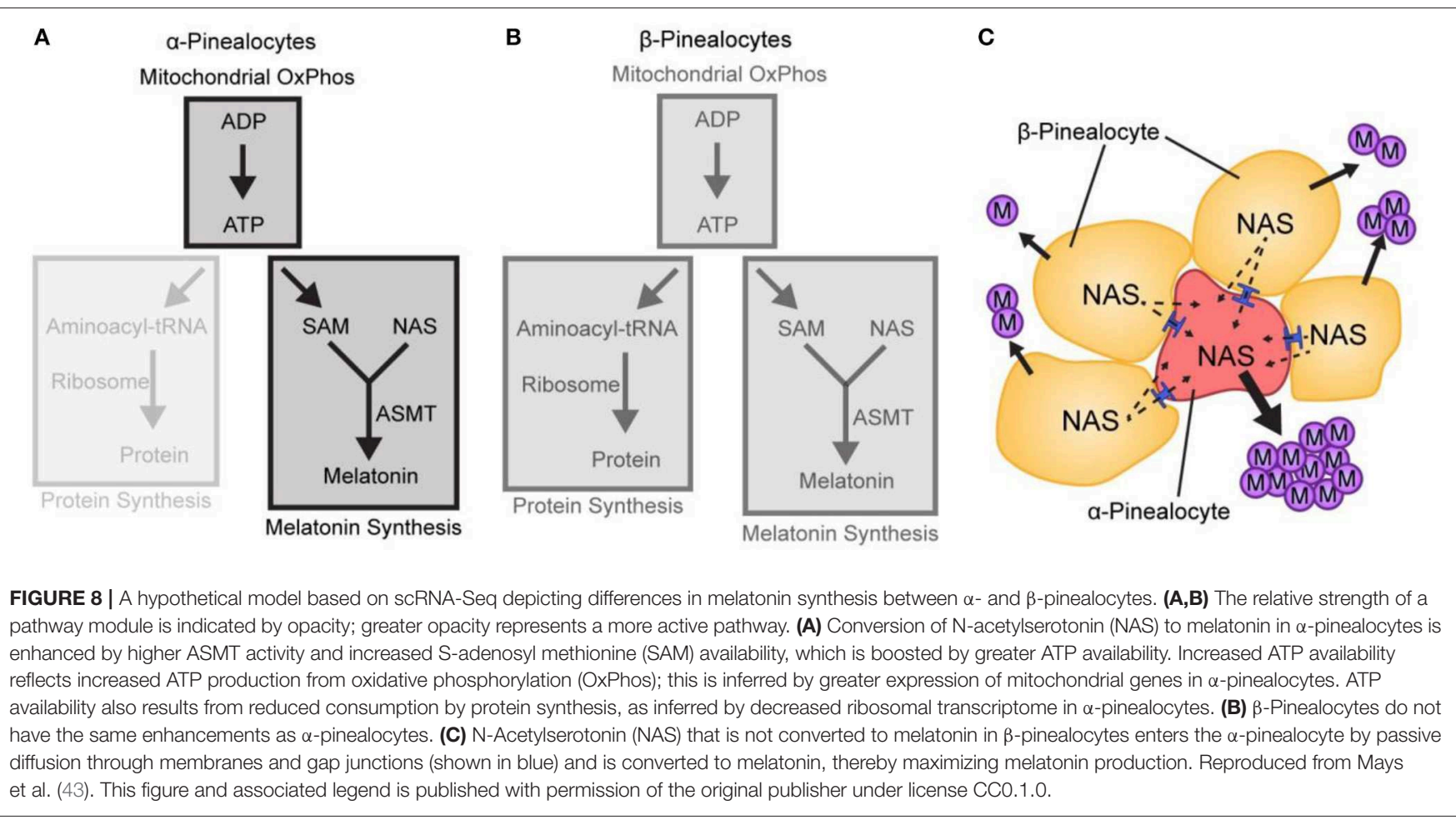

seems possible that such changes could support the seemingly compromised $\alpha$-pinealocytes.

A final issue to be addressed is how the number of each of the pinealocyte subtypes is regulated. One hypothetical possibility is that distinct phenotypes develop early in ontogeny and the cells cannot undergo a shift from one subtype to another. In this scenario, the phenotypes are not reversible; their relative abundance might only reflect selective cell death and replacement. A second hypothetical possibility is that $\alpha$ and $\beta$-pinealocyte phenotypes are reversible and can shift back and forth at any time during development and maturity. The controlling factor or factors could be circulating in nature or reflect neural stimulation, perhaps influenced by the day length. This hypothetical reversible mechanism might fine tune melatonin production.

\section{CONCLUDING COMMENT}

The work reviewed here is impressive in documenting how methods have evolved from requiring a few milligrams of tissue to document a single transcript to documenting thousands of transcripts in a single cell!

scRNA-seq establishes a new foundation for research on pineal cell biology by introducing new methods and concepts and by segregating gene expression into separate cells. Moreover, it has reshaped our thinking about the pineal gland by adding to the complex nature of the tissue, by providing transcriptionally defined cell types. The work is unique in that two states of physiological activity-day and night-are characterized, which adds another dimension to the value of scRNA-seq of this tissue.
Work on the pineal gland has the potential to improve our understanding of the basic mechanisms that underlie the function of this tissue in non-human primates and humans. Bulk sequencing of the rhesus pineal gland indicates that there are fundamental differences between it and the rat, as regards day/night changes in transcript abundance $(22,55)$. It will be of interest to use scRNA-seq technology to learn more about the human and rhesus pineal glands, with the intention of understanding how cells in this tissue communicate and are regulated.

One avenue that will challenge investigators is the analysis of isoform regulation (23) on a single cell basis, with the goal of understanding the association of specific isoforms with cell types and how they are regulated. The discoveries revealed by scRNAseq and advanced forms of sequencing will shape future studies on pineal cell biology.

\section{AUTHOR CONTRIBUTIONS}

SC, CF, SH, LH, JMa, MCK, MWK, JMu, MR, LS, and DK: substantial contributions to the conception or design of the work, or the acquisition, analysis, or interpretation of data for the work. SC, SH, LH, JMu, MCK, MWK, JMa, MR, and DK: drafting the work or revising it critically for important intellectual content. SC, LH, SH, MWK, JMa, MR, JMu, MCK, MR, and DK: provided approval for publication of the content.

\section{FUNDING}

This work was supported by the Intramural Research Program, National Human Genome Research Institute, National Institutes 
of Health, Bethesda, MD 20892 (to SH and JMu) (https:// www.genome.gov) for work on the transcriptome of the rat pineal gland; the Intramural Research Program of the National Institute on Deafness and Other Communication Disorders (to JMa, MCK, and MWK) for work on rat pineal single cell isolation and RNA sequencing (https://www.nidcd. nih.gov/), the Eunice Kennedy Shriver National Institute of Child Health and Human Development (to LH, SC, CF, LS, MR, and DK) for animals, immunohistochemistry, cell isolation, and cell analysis. MR received support from Nordisk Foundation (http://novonordiskfonden.dk/en), Lundbeck Foundation (grant R108-A10301, https://www. lundbeckfonden.com/en/), Carlsberg Foundation (grants CF150515 and CF17-0070, http://www.carlsbergfondet.dk/en), and from the Independent Research Fund of Denmark (grant 8020-000378, https://dff.dk/en) for immunohistochemical studies. The funders had no role in study design, data

\section{REFERENCES}

1. Moller M, Baeres FM. The anatomy and innervation of the mammalian pineal gland. Cell Tissue Res. (2002) 309:139-50. doi: 10.1007/s00441-002-0580-5

2. Darmon MC, Guibert B, Leviel V, Ehret M, Maitre M, Mallet J. Sequence of two mRNAs encoding active rat tryptophan hydroxylase. J Neurochem. (1988) 51:312-6. doi: 10.1111/j.1471-4159.1988.tb04871.x

3. Grenett HE, Ledley FD, Reed LL, Woo SL. Full-length cDNA for rabbit tryptophan hydroxylase: functional domains and evolution of aromatic amino acid hydroxylases. Proc Natl Acad Sci USA. (1987) 84:55304. doi: $10.1073 /$ pnas.84.16.5530

4. Ishida I, Obinata M, Deguchi T. Molecular cloning and nucleotide sequence of cDNA encoding hydroxyindole O-methyltransferase of bovine pineal glands. J Biol Chem. (1987) 262:2895-9.

5. Dumas S, Darmon MC, Delort J, Mallet J. Differential control of tryptophan hydroxylase expression in raphe and in pineal gland: evidence for a role of translation efficiency. J Neurosci Res. (1989) 24:53747. doi: 10.1002/jnr.490240412

6. Hirayama K, Lentz SI, Kapatos G. Tetrahydrobiopterin cofactor biosynthesis: GTP cyclohydrolase I mRNA expression in rat brain and superior cervical ganglia. J Neurochem. (1993) 61:1006-14. doi: 10.1111/j.1471-4159.1993.tb03614.x

7. Kutty RK, Kutty G, Duncan T, Nickerson J, Chader GJ, Wiggert B. Radioanalytic estimation of amplification products generated by reverse transcription PCR using [alpha-33P] deoxyribonucleoside triphosphate. Biotechniques. (1993) 15:808, 811-2.

8. Mato E, Santisteban P, Viader M, Capella G, Fornas O, Puig-Domingo M, et al. Expression of somatostatin in rat pineal cells in culture. J Pineal Res. (1993) 15:43-5. doi: 10.1111/j.1600-079X.1993.tb00508.x

9. Craft CM, Whitmore DH, Wiechmann AF. Cone arrestin identified by targeting expression of a functional family. J Biol Chem. (1994) 269:4613-9.

10. Olcese J, Muller D, Munker M, Schmidt C. Natriuretic peptides elevate cyclic $3^{\prime}, 5^{\prime}$-guanosine monophosphate levels in cultured rat pinealocytes: evidence for guanylate cyclase-linked membrane receptors. Mol Cell Endocrinol. (1994) 103:95-100. doi: 10.1016/0303-7207(94)90074-4

11. Gauer F, Kedzierski W, Craft CM. Identification of circadian gene expression in the rat pineal gland and retina by mRNA differential display. Neurosci Lett. (1995) 187:69-73. doi: 10.1016/0304-3940(95)11331-P

12. Reppert SM, Godson C, Mahle CD, Weaver DR, Slaugenhaupt SA, Gusella JF. Molecular characterization of a second melatonin receptor expressed in human retina and brain: the Mellb melatonin receptor. Proc Natl Acad Sci USA. (1995) 92:8734-8. doi: 10.1073/pnas.92.19.8734

13. Schaad NC, Vanecek J, Rodriguez IR, Klein DC, Holtzclaw L, Russell JT. Vasoactive intestinal peptide elevates pinealocyte intracellular calcium collection and analysis, decision to publish, or preparation of the manuscript.

\section{ACKNOWLEDGMENTS}

The assistance of the following members of the National Institutes of Health Intramural Sequencing Center Comparative Sequencing Program is appreciated: Betty Barnabas, MPH, MSc; Robert Blakesley, PhD; Gerry Bouffard, PhD; Shelise Brooks, BS; Holly Coleman, MSc; Mila Dekhtyar, MSc; Michael Gregory, MSc; Xiaobin Guan, PhD; Joel Han, BS; Shi-ling Ho, BS; Richelle Legaspi, MSc; Quino Maduro, BS; Cathy Masiello, MSc; Jenny McDowell, PhD; Casandra Montemayor, MSc; James Mullikin, PhD; Morgan Park, PhD; Nancy Riebow, BS; Karen Schandler, MSc; Brian Schmidt, BS; Christina Sison, BS; Mal Stantripop, BS; James Thomas, PhD; Pam Thomas, PhD; Meg Vemulapalli, MSc; Alice Young, BA. concentrations by enhancing influx: evidence for involvement of a cyclic GMP-dependent mechanism. Mol Pharmacol. (1995) 47:923-33.

14. Humphries A, Klein D, Baler R, Carter DA, cDNA array analysis of pineal gene expression reveals circadian rhythmicity of the dominant negative helixloop-helix protein-encoding gene, Id-1. J Neuroendocrinol. (2002) 14:1018. doi: 10.1046/j.0007-1331.2001.00738.x

15. Bailey MJ, Beremand PD, Hammer R, Reidel E, Thomas TL, Cassone VM. Transcriptional profiling of circadian patterns of mRNA expression in the chick retina. J Biol Chem. (2004) 279:52247-54. doi: 10.1074/jbc.M405 679200

16. Bailey MJ, Beremand PD, Hammer R, Bell-Pedersen D, Thomas TL, Cassone VM. Transcriptional profiling of the chick pineal gland, a photoreceptive circadian oscillator and pacemaker. Mol Endocrinol. (2003) 17:208495. doi: 10.1210/me.2003-0121

17. Munoz EM, Bailey MJ, Rath MF, Shi Q, Morin F, Coon SL, et al. NeuroD1: developmental expression and regulated genes in the rodent pineal gland. $J$ Neurochem. (2007) 102:887-99. doi: 10.1111/j.1471-4159.2007.04605.x

18. Kim JS, Bailey MJ, Ho AK, Moller M, Gaildrat P, Klein DC. Daily rhythm in pineal phosphodiesterase (PDE):activity reflects adrenergic $/ 3^{\prime}, 5^{\prime}$ cyclic adenosine $5^{\prime}$-monophosphate induction of the PDE4B2 variant. Endocrinology. (2007) 148:1475-85. doi: 10.1210/en.2006-1420

19. Bailey MJ, Coon SL, Carter DA, Humphries A, Kim JS, Shi Q, et al. Night/day changes in pineal expression of $>600$ genes: central role of adrenergic/cAMP signaling. J Biol Chem. (2009) 284:7606-22. doi: 10.1074/jbc.M808394200

20. Toyama R, Chen X, Jhawar N, Aamar E, Epstein J, Reany N, et al. Transcriptome analysis of the zebrafish pineal gland. Dev Dyn. (2009) 238:1813-26. doi: 10.1002/dvdy.21988

21. Coon SL, Munson PJ, Cherukuri PF, Sugden D, Rath MF, Moller M, et al. Circadian changes in long noncoding RNAs in the pineal gland. Proc Natl Acad Sci USA. (2012) 109:13319-24. doi: 10.1073/pnas.1207748109

22. Backlund PS, Urbanski HF, Doll MA, Hein DW, Bozinoski M, Mason CE, et al. Daily rhythm in plasma N-acetyltryptamine. J Biol Rhythms. (2017) 32:195-211. doi: 10.1177/0748730417700458

23. Hartley SW, Mullikin JC, Klein DC, Park M, NISC Comparative Sequencing Program, Coon SL. Alternative isoform analysis of Ttc8 expression in the rat pineal gland using a multi-platform sequencing approach reveals neural regulation. PLoS ONE. (2016) 11:e0163590. doi: 10.1371/journal.pone.0163590

24. Yamazaki F, Moller M, Fu C, Clokie SJ, Zykovich A, Coon SL, et al. The Lhx9 homeobox gene controls pineal gland development and prevents postnatal hydrocephalus. Brain Struct Funct. (2015) 220:1497509. doi: 10.1007/s00429-014-0740-x

25. Hartley SW, Coon SL, Savastano LE, Mullikin JC, NISC Comparative Sequencing Program, Fu C, et al. Neurotranscriptomics: the effects of neonatal 
stimulus deprivation on the rat pineal transcriptome. PLoS ONE. (2015) 10:e0137548. doi: 10.1371/journal.pone. 0137548

26. Matsuo M, Coon SL, Klein DC. RGS2 is a feedback inhibitor of melatonin production in the pineal gland. FEBS Lett. (2013) 587:13928. doi: 10.1016/j.febslet.2013.03.016

27. Tovin A, Alon S, Ben-Moshe Z, Mracek P, Vatine G, Foulkes NS, et al. Systematic identification of rhythmic genes reveals camklgb as a new element in the circadian clockwork. PLoS Genet. (2012) 8:e1003116. doi: 10.1371/journal.pgen. 1003116

28. Ochocinska MJ, Munoz EM, Veleri S, Weller JL, Coon SL, Pozdeyev N, et al. NeuroD1 is required for survival of photoreceptors but not pinealocytes: results from targeted gene deletion studies. J Neurochem. (2012) 123:4459. doi: 10.1111/j.1471-4159.2012.07870.x

29. Clokie SJ, Lau P, Kim HH, Coon SL, Klein DC. MicroRNAs in the pineal gland: miR-483 regulates melatonin synthesis by targeting arylalkylamine N-acetyltransferase. J Biol Chem. (2012) 287:25312-24. doi: 10.1074/jbc.M112.356733

30. Rovsing L, Clokie S, Bustos DM, Rohde K, Coon SL, Litman T, et al. Crx broadly modulates the pineal transcriptome. J Neurochem. (2011) 119:26274. doi: 10.1111/j.1471-4159.2011.07405.x

31. Yamazaki F, Kim HH, Lau P, Hwang CK, Iuvone PM, Klein D, et al. pY RNA1s2: a highly retina-enriched small RNA that selectively binds to Matrin 3 (Matr3). PLoS ONE. (2014) 9:e88217. doi: 10.1371/journal.pone.0088217

32. Riazuddin SA, Iqbal $M$, Wang $Y$, Masuda $T$, Chen $Y$, Bowne $S$, et al. A splice-site mutation in a retina-specific exon of BBS8 causes nonsyndromic retinitis pigmentosa. Am J Hum Genet. (2010) 86:80512. doi: 10.1016/j.ajhg.2010.04.001

33. Murphy D, Singh R, Kolandaivelu S, Ramamurthy V, Stoilov P. Alternative splicing shapes the phenotype of a mutation in BBS8 to cause nonsyndromic retinitis pigmentosa. Mol Cell Biol. (2015) 35:1860-70. doi: 10.1128/MCB.00040-15

34. Ansley SJ, Badano JL, Blacque OE, Hill J, Hoskins BE, Leitch CC, et al. Basal body dysfunction is a likely cause of pleiotropic Bardet-Biedl syndrome. Nature. (2003) 425:628-33. doi: 10.1038/nature02030

35. Bin J, Madhavan J, Ferrini W, Mok CA, Billingsley G, Heon E. BBS7 and TTC8 (BBS8) mutations play a minor role in the mutational load of BardetBiedl syndrome in a multiethnic population. Hum Mutat. (2009) 30:E73746. doi: 10.1002/humu. 21040

36. Nawy T. Single-cell epigenetics. Nat Methods. (2013) 10:1060. doi: 10.1038/nmeth. 2721

37. Rath MF, Coon SL, Amaral FG, Weller JL, Moller M, Klein DC. Melatonin synthesis: acetylserotonin O-methyltransferase (ASMT) is strongly expressed in a subpopulation of pinealocytes in the male rat pineal gland. Endocrinology. (2016) 157:2028-40. doi: 10.1210/en.2015-1888

38. Buda M, Klein DC. A suspension culture of pinealocytes: regulation of $\mathrm{N}$-acetyltransferase activity. Endocrinology. (1978) 103:1483-93. doi: 10.1210/endo-103-4-1483

39. Vanecek J, Sugden D, Weller J, Klein DC. Atypical synergistic alpha 1- and beta-adrenergic regulation of adenosine $3^{\prime}, 5^{\prime}$-monophosphate and guanosine $3^{\prime}, 5^{\prime}$-monophosphate in rat pinealocytes. Endocrinology. (1985) 116:216773. doi: 10.1210/endo-116-6-2167

40. Sugden D, Vanecek J, Klein DC, Thomas TP, Anderson WB. Activation of protein kinase $\mathrm{C}$ potentiates isoprenaline-induced cyclic AMP accumulation in rat pinealocytes. Nature. (1985) 314:359-61. doi: 10.1038/314 $359 \mathrm{a} 0$

41. Cena V, Halperin JI, Yeandle S, Klein DC. Norepinephrine stimulates potassium efflux from pinealocytes: evidence for involvement of biochemical "AND" gate operated by calcium and adenosine $3^{\prime}, 5^{\prime}$-monophosphate. Endocrinology. (1991) 128:559-69. doi: 10.1210/endo-128-1-559
42. Ganguly S, Grodzki C, Sugden D, Moller M, Odom S, Gaildrat P, et al. Neural adrenergic/cyclic AMP regulation of the immunoglobulin E receptor alpha-subunit expression in the mammalian pinealocyte: a neuroendocrine/immune response link? J Biol Chem. (2007) 282:3275864. doi: $10.1074 /$ jbc.M705950200

43. Mays JC, Kelly MC, Coon SL, Holtzclaw L, Rath MF, Kelley MW, et al. Singlecell RNA sequencing of the mammalian pineal gland identifies two pinealocyte subtypes and cell type-specific daily patterns of gene expression. PLoS ONE. (2018) 13:e0205883. doi: 10.1371/journal.pone.0205883

44. Pevet P. On the presence of different populations of pinealocytes in the mammalian pineal gland. J Neural Transm. (1977) 40:289304. doi: 10.1007/BF01257021

45. Zeisel A, Munoz-Manchado AB, Codeluppi S, Lonnerberg P, La Manno $\mathrm{G}$, Jureus A, et al. Brain structure. Cell types in the mouse cortex and hippocampus revealed by single-cell RNA-seq. Science. (2015) 347:113842. doi: $10.1126 /$ science.aaa1934

46. Zhong S, Zhang S, Fan X, Wu Q, Yan L, Dong J, et al. A single-cell RNAseq survey of the developmental landscape of the human prefrontal cortex. Nature. (2018) 555:524-528. doi: 10.1038/nature25980

47. Rosenberg AB, Roco CM, Muscat RA, Kuchina A, Sample P, Yao Z, et al. Single-cell profiling of the developing mouse brain and spinal cord with splitpool barcoding. Science. (2018) 360:176-82. doi: 10.1126/science.aam8999

48. Lopez-Munoz F, Calvo JL, Boya J, Carbonell AL. Coexpression of vimentin and glial fibrillary acidic protein in glial cells of the adult rat pineal gland. $J$ Pineal Res. (1992) 12:145-8. doi: 10.1111/j.1600-079X.1992.tb00041.x

49. Suzuki T, Kachi T. Immunohistochemical studies on supporting cells in the adrenal medulla and pineal gland of adult rat, especially on S-100 protein, glial fibrillary acidic protein and vimentin. Kaibogaku Zasshi. (1995) 70:130-9.

50. Zang X, Nilaver G, Stein BM, Fetell MR, Duffy PE. Immunocytochemistry of pineal astrocytes: species differences and functional implications. J Neuropathol Exp Neurol. (1985) 44:486-95. doi: 10.1097/00005072-198509000-00004

51. Raj B, Wagner DE, McKenna A, Pandey S, Klein AM, Shendure J, et al. Simultaneous single-cell profiling of lineages and cell types in the vertebrate brain. Nat Biotechnol. (2018) 36:442-50. doi: 10.1038/nbt.4103

52. Ranasinghe C, Trivedi S, Wijesundara DK, Jackson RJ. IL-4 and IL-13 receptors: roles in immunity and powerful vaccine adjuvants. Cytokine Growth Factor Rev. (2014) 25:437-42. doi: 10.1016/j.cytogfr.2014.07.010

53. Kania A, Klein R. Mechanisms of ephrin-Eph signalling in development, physiology and disease. Nat Rev Mol Cell Biol. (2016) 17:240-56. doi: 10.1038/nrm.2015.16

54. Parfitt AG, Klein DC. Sympathetic nerve endings in the pineal gland protect against acute stress-induced increase in $\mathrm{N}$-acetyltransferase (EC 2.3.1.5.) activity. Endocrinology. (1976) 99:840-51. doi: 10.1210/endo-99-3-840

55. Coon SL, Del Olmo E, Young WS III, Klein DC. Melatonin synthesis enzymes in Macaca mulatta: focus on arylalkylamine N-acetyltransferase (EC 2.3.1.87). J Clin Endocrinol Metab. (2002) 87:4699-706. doi: 10.1210/jc.2002-020683

Conflict of Interest Statement: The authors declare that the research was conducted in the absence of any commercial or financial relationships that could be construed as a potential conflict of interest.

Copyright (๑) 2019 Coon, Fu, Hartley, Holtzclaw, Mays, Kelly, Kelley, Mullikin, Rath, Savastano and Klein. This is an open-access article distributed under the terms of the Creative Commons Attribution License (CC BY). The use, distribution or reproduction in other forums is permitted, provided the original author(s) and the copyright owner(s) are credited and that the original publication in this journal is cited, in accordance with accepted academic practice. No use, distribution or reproduction is permitted which does not comply with these terms. 